\title{
Observing Techniques for Astronomical Laser Guide Star Adaptive Optics
}

\author{
C.E. Max
}

B. Macintosh

S.S. Olivier

D.T. Gavel

H.W. Friedman

This paper was prepared for submittal to the Society of Photo-Optical Instrumentation Engineers Conference Kona, $\mathrm{HI}$

March 22-27, 1998

May 1998

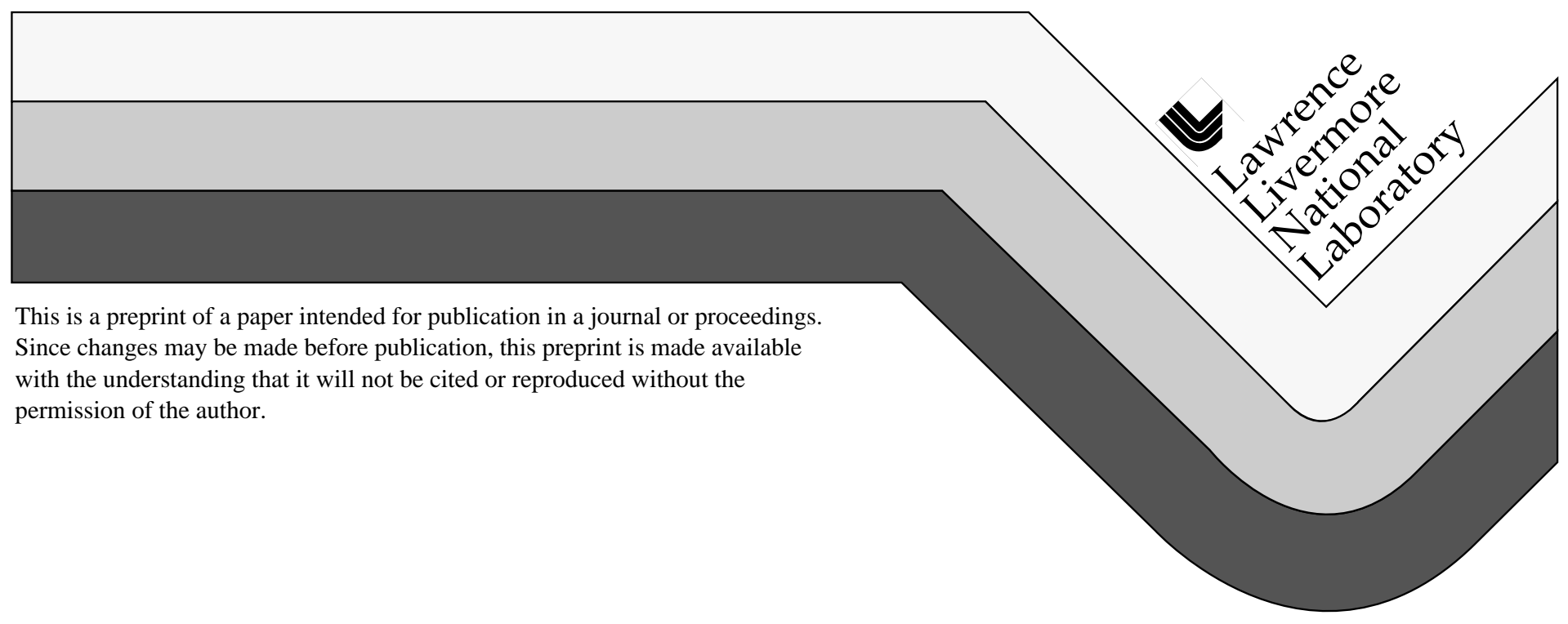




\section{DISCLAIMER}

This document was prepared as an account of work sponsored by an agency of the United States Government. Neither the United States Government nor the University of California nor any of their employees, makes any warranty, express or implied, or assumes any legal liability or responsibility for the accuracy, completeness, or usefulness of any information, apparatus, product, or process

disclosed, or represents that its use would not infringe privately owned rights. Reference herein to any specific commercial product, process, or service by trade name, trademark, manufacturer, or otherwise, does not necessarily constitute or imply its endorsement, recommendation, or favoring by the United States Government or the University of California. The views and opinions of authors expressed herein do not necessarily state or reflect those of the United States Government or the University of California, and shall not be used for advertising or product endorsement purposes. 


\title{
Observing Techniques for Astronomical Laser Guide Star Adaptive Optics
}

\author{
C. E. Max ${ }^{\mathrm{a}}$, B. Macintosh, S. S. Olivier, D. T. Gavel, and H. W. Friedman \\ Lawrence Livermore National Laboratory, Livermore, CA 94550
}

\begin{abstract}
We discuss astronomical observing requirements and their implementation using sodium-layer laser guide star adaptive optics. Specific issues requiring implementation include the ability to place the astronomical object at different locations within the field of view; reliable subtraction of Rayleigh-scattered light; efficient focusing; and stable point-spread-function characterization.
\end{abstract}

Keywords: adaptive optics, laser guide stars, point spread function

\section{INTRODUCTION}

Sodium-layer laser guide stars have been demonstrated to provide an acceptable wavefront reference for astronomical adaptive optics systems $^{1,2}$. However the implementation of effective sodium-layer laser guide star systems for astronomical telescopes will require attention to specific observing requirements and constraints. "Nodding" requires the astronomical object and the laser spot to be placed at arbitrary positions within the field of view. The coupled laser control system and adaptive optics system must be able to efficiently subtract Rayleigh scattered light from the wavefront sensor subapertures, so that Rayleigh scattering does not bias the centroid positions. Focusing the laser launch telescope, the main telescope, and the wavefront sensor must be done efficiently, in the face of time-dependent sodium-layer height. And most important of all, the point spread function of the entire system must be quantitatively characterized in order to enable astrophysically meaningful data to be obtained.

\section{LOCATION WITHIN THE ADAPTIVE OPTICS FIELD OF VIEW}

A common observing mode for natural guide star adaptive optics places a bright reference star off the axis of the optical system, so that a faint astronomical target object can be placed on-axis. For laser guide star observations, on the other hand, the laser can usually be pointed directly at the on-axis astronomical target, because the laser light can be screened out spectrally. The tip-tilt sensor will be pointed at the astronomical target if the latter is point-like and sufficiently bright. If not, the tip-tilt sensor will be pointed at an off-axis tilt reference star, up to an arc minute away.

For infra-red observations in both natural guide star mode and laser guide star mode, it is desirable to be able to "nod" the telescope. The purpose of these small telescope "nods" or offsets is to move the astronomical object and the backgrounds from sky and telescope to different locations on the infra-red array, so as to enable precision background subtraction. The telescope offsets required for nodding range from a few arc seconds up to perhaps half an arc minute, for most adaptive optics observing scenarios.

To provide the flexibility needed for selectable reference star location and for nodding, a controllable optical arrangement such as a pair of steering mirrors in front of the wavefront sensor ${ }^{3}$ is highly desirable. In laser guide star mode, there must be an analogous capability to "counter-nod" the laser pointing optics so that the adaptive optics control loop remains closed during nodding observations.

\section{SUBTRACTING RAYLEIGH SCATTERED LIGHT}

Although the sodium-layer laser guide star is relatively bright (e.g. $8^{\mathrm{m}}$ to $12^{\mathrm{m}}$ equivalent), light that is Rayleigh scattered by molecular oxygen, nitrogen, and aerosols low in the atmosphere (at altitudes of $\sim 35 \mathrm{~km}$ and below) will typically be many times more intense than the light returning from the sodium layer (recall the $\mathrm{r}^{-4}$ dependence of the Radar Equation). An iris can be used in front of the wavefront sensor to block most Rayleigh light. Both directly viewed Rayleigh light and Rayleigh

\footnotetext{
${ }^{\mathrm{a}}$ Further author information - email addresses

C.E.M. max1@1lnl.gov B.M. bmac@igpp.llnl.gov

D.T.G. gavel1@1lnl.gov H.W.F. friedman3@1lnl.gov

S.S.O. olivier1@1lnl.gov
} 
light that has been scattered by elements of the telescope and optical system can also be subtracted from the guide star images in each wavefront sensor subaperture, in order to minimize wavefront errors. The viewing geometry is illustrated in Figure 1.

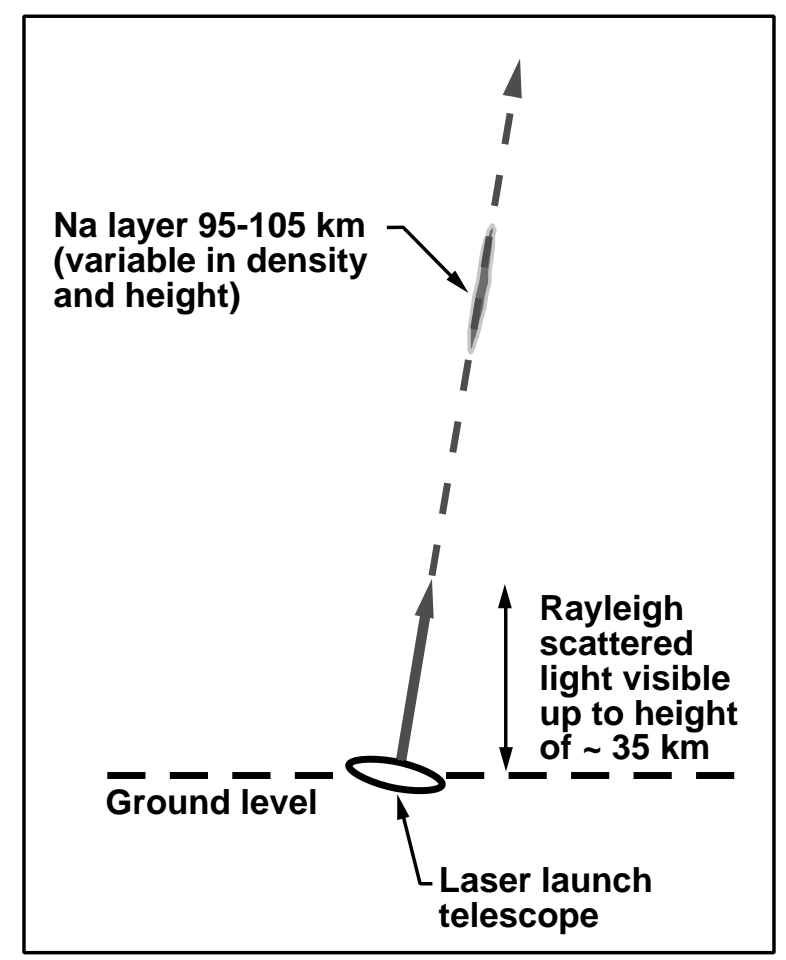

Figure 1. Schematic features of sodium-layer laser guide star generation.

Tunable lasers (e.g. dye lasers or Raman-shifting lasers) can use frequency shifting to subtract the Rayleigh scattered light. This procedure takes advantage of the fact that when the laser is tuned to the frequency of the sodium $\mathrm{D}_{2}$ resonance line at 589 $\mathrm{nm}$, both Rayleigh scattered light and sodium-layer resonance-scattered light appear in the wavefront sensor. However if the laser is tuned several GHz off the $\mathrm{D}_{2}$ resonance line, only Rayleigh scattering will be seen. Figure 2 illustrates the guide-star regions as seen by the telescope guide camera. For calibration, the wavefront-sensor equivalent of Figure $2 \mathrm{~b}$ can be used as a "background" frame, in order to avoid biasing the centroid calculation by the presence of Rayleigh scattered light. The laser control system implements this procedure on command.
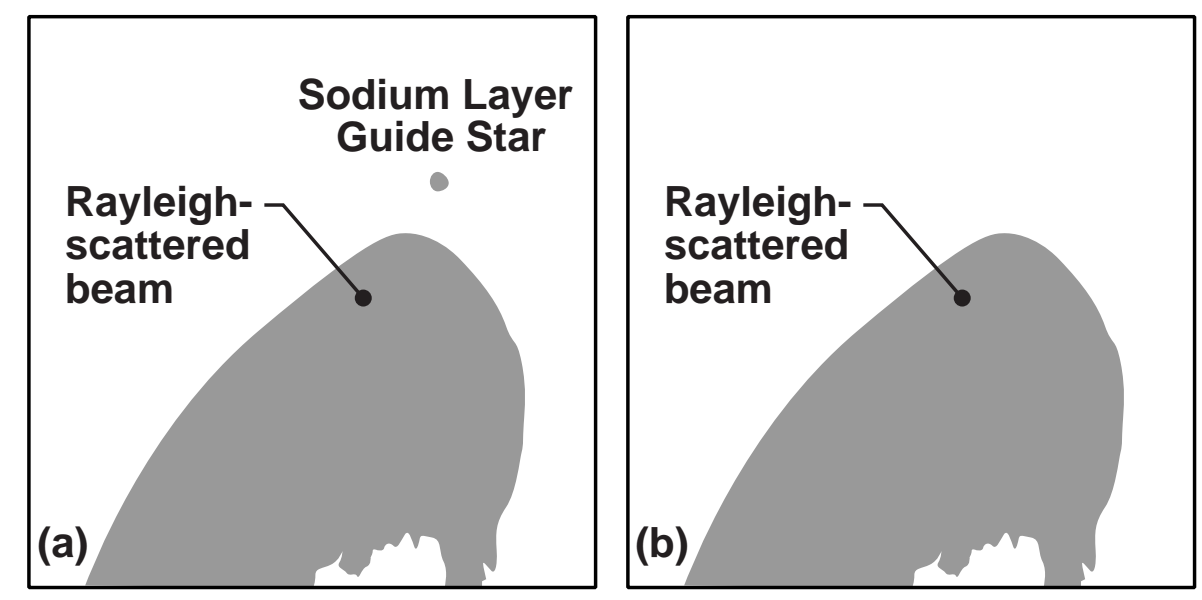

Figure 2. a) Sodium-layer laser guide star (top) and Rayleigh scattered light (bottom) as seen through guide camera on telescope. b) Rayleigh scattered light only (laser is tuned $3 \mathrm{GHz}$ off central frequency of $\mathrm{D}_{2}$ line). 
Time-gating provides an alternate technique for eliminating Rayleigh scatter from the wavefront sensor. This concept requires a pulsed laser, and involves "shuttering" the wavefront sensor so that it is not sensitive to light when the laser pulse is propagating in the lower part of the atmosphere where Rayleigh scattering is dominant. The sum-frequency laser developed by Lincoln Laboratories has a pulse format that is amenable to this methodology ${ }^{4}$.

\section{FOCUSING EFFICIENTLY DURING LASER GUIDE STAR OPERATION}

Maintaining good telescope focus is already a challenge at many astronomical observatories. A variety of automated focusing procedures have been developed to minimize the time lost to repeated refocusing exercises, as both the temperature and telescope elevation angle change during the night.

Sodium laser guide stars add several additional complexities to the issue of telescope focusing. The height distribution of atomic sodium in the mesospheric sodium layer changes during the night, on timescales of minutes to hours. In addition, the geometric distance to the sodium layer depends on telescope elevation angle. In the face of these variations, both the wavefront sensor and the laser launch telescope must remain focused on the peak of the sodium-layer density, while the main telescope remains well focused at infinity.

Adaptive optics systems have many points at which focus corrections can be introduced. The first issue is to decide which one is most suitable for maintaining focus on the sodium layer. One straightforward method is to control the position of the wavefront sensor focus stage, in response to a slow focus sensor which observes a natural star. Output from the slow focus sensor can also be fed back to the laser control system, which maintains the focus of the laser launch telescope.

Initialization of such a system at the beginning of each night may need to be done manually, and can be tedious. Currently with the sodium-layer laser guide star system at Lick Observatory, we perform the initial focus and the calibration for Rayleigh scattered light in the following manner:

a) Focus the main telescope on a natural star (focus at infinity).

b) Propagate the laser through its launch telescope, aimed at the natural star.

c) Move the secondary mirror of the astronomical telescope to focus on the sodium layer as well as possible, and observe the sodium star on the telescope's guide camera at high resolution. Use the guide camera image to focus the laser launch telescope and obtain the smallest laser guide star image.

d) Once the laser launch telescope has been focused on the sodium layer, re-focus the main telescope at infinity. Move the guider off-axis so that the laser guide star light now reaches the wavefront sensor.

e) Stop down the iris in front of the wavefront sensor to block the Rayleigh scattered light as much as possible. Tune the laser on and off central wavelength for Rayleigh background subtraction.

f) Measure the amount of focus on the laser guide star using the Hartmann wavefront sensor centroid positions.

g) Feed back this focus information to move the wavefront sensor stage to the position of best focus.

Steps f) and g) can be replaced by use of a separate slow focus sensor, once the initial system focus has been defined.

\section{CHARACTERIZING THE POINT SPREAD FUNCTION OF A SODIUM-LAYER LASER GUIDE STAR ADAPTIVE OPTICS SYSTEM}

Astronomical applications require knowledge of the point spread function produced by the adaptive optics system during the whole time-integration on the astronomical object of interest. This is crucial for quantitative photometry (for example to study variability or to derive colors), for spectroscopy, and in order to quantify the ability to detect faint objects close to bright objects (for example to measure faint stellar companions, to detect planets, or to characterize faint nebulosity near a bright star).

Each of these applications typically uses deconvolution methods of data analysis, to derive quantitative astrophysical conclusions. One class of deconvolution techniques, "blind iterative deconvolution", deduces both the point spread function and the image details from image data alone. However it is currently more common to have an independent measure of the point spread function. The usual method for obtaining the point spread function involves integrating on an astronomical object for a period of time; then integrating on a point source to characterize its shape; then returning to the astronomical object of interest; and repeating this process. 
For natural guide star adaptive optics this methodology is already problematical: the atmospheric turbulence characteristics change on a timescale of minutes; the point reference source needs to be the same magnitude and color as the adaptive optics reference in order for the adaptive optics system performance to be comparable; and the method is quite time-consuming. Moreover, it can be argued that such a methodology is not practical at all for a laser guide star, for several reasons:

- Because of tilt anisoplanatism, the point spread function would have to be measured using a binary tip-tilt star whose primary component had the same magnitude and color as the original tip-tilt star, and which had a faint companion star to serve as the surrogate astronomical point-source. The laser guide star would be pointed directly at the faint companion star in order to close the adaptive optics loop.

- The tip-tilt star and its point source companion would have to have the same angular separation as the original astronomical target and its tip-tilt reference star.

- The faint companion star would have to be sufficiently faint that it did not appear in the wavefront sensor. Alternatively one would have to use a notch filter in front of the wavefront sensor to admit only laser light. (One would like to avoid the latter, since narrow-band filters attenuate the light outside the bandpass, and would thus decrease the signal to the wavefront sensor.)

A major difficulty in implementing this type of direct point spread function characterization is that available star catalogues do not contain sufficient information to identify suitable binary star pairs, which for an 8 to 10 meter telescope are typically $17^{\mathrm{m}}-19^{\mathrm{m}}$ when used for tip-tilt reference. The advent of the new generation of surveys (such as the Sloan Digital Sky Survey) will help ameliorate this situation, but the latter are not expected to be complete for several years.

Veran and colleagues ${ }^{6}$ have implemented a technique for estimating the PSF for natural guide star adaptive optics, which does not require frequent pointing away from the astronomical target. In this technique the adaptive optics wavefront sensor error signals and the deformable mirror voltages provide on-line information to reconstruct the adaptive optics PSF during the course of a long astronomical observation. The wavefront sensor directly measures the wavefront errors at spatial frequencies less than the inverse of the subaperture spacing, or for angles less than the observing wavelength divided by the subaperture spacing. The deformable mirror voltages provide the corrected wavefronts, which at low spatial frequencies are approximately equal to the actual wavefronts incident on the telescope. From the deformable mirror voltages one can get a good estimate of $\mathrm{r}_{0}$, the atmospheric coherence parameter. Knowing $\mathrm{r}_{0}$, we can statistically characterize the higher spatial frequencies (angles larger than the observing wavelength divided by the subaperture spacing). Data from $\mathrm{CFHT}^{6}$ show that this technique works well for reference stars brighter than about 14th magnitude.

An analogous technique could be developed for use with laser guide stars. In principle Veran's analysis applies for laser guide stars as well as natural guide stars, with two important exceptions:

1) Tip-tilt information can no longer be obtained from the wavefront sensor. It will be necessary to keep additional records concerning tip-tilt control loop operation: voltages sent to the tip-tilt mirror, and tip-tilt sensor centroid signals, in order to characterize the real-time performance of the tip-tilt loop.

2) Focus anisoplanatism, or the "cone effect", needs to be characterized independently. This might be done, for example, by using SCIDAR to measure $\mathrm{C}_{\mathrm{n}}{ }^{2}$ as a function of altitude, and then calculating the predicted focus anisoplanatism assuming expressions derived from Kolmogorov turbulence ${ }^{7}$. Today very little quantitative information exists concerning focus anisoplanatism under realistic observing conditions at astronomical sites. It may turn out that at a given telescope there is little variability in the size of the focus anisoplanatism, in which case real-time measurements would not be needed. However much more data are needed before such a conclusion can be reached.

\section{CONCLUSIONS}

\section{ACKNOWLEDGMENTS}

This research was performed at the Lawrence Livermore National Laboratory under the auspices of US Department of Energy Contract number W-7405-ENG-48. 


\section{REFERENCES}

1. W. Happer, G. MacDonald, C. E. Max, and F. Dyson, J. Opt. Soc. Am. A 11, 263 (1994).

2. C. E. Max et al., Science 277, 1649 (1997).

3. T. Gregory et al., Opt. Soc. Am. Tech. Digest Series 13, 11 (1996).

4. T. H. Jeys, A. A. Brailove, and A. Mooradian, Appl. Opt. 28, 2588 (1989).

5. J. C. Christou, D. Bonaccini, and N. Ageorges, Proc. SPIE 3126, 68 (1997); J. C. Christou, Proc. SPIE 2534, 226 (1995).

6. J. P. Veran, F. J. Rigaut, D. Rouan, and H. Maitre, J. Opt. Soc. Am. A 14, 3057 (1997).

7. R. J. Sasiela, Electromagnetic Wave Propagation in Turbulence (Springer-Verlag, Berlin, 1994), pages 150-160. 


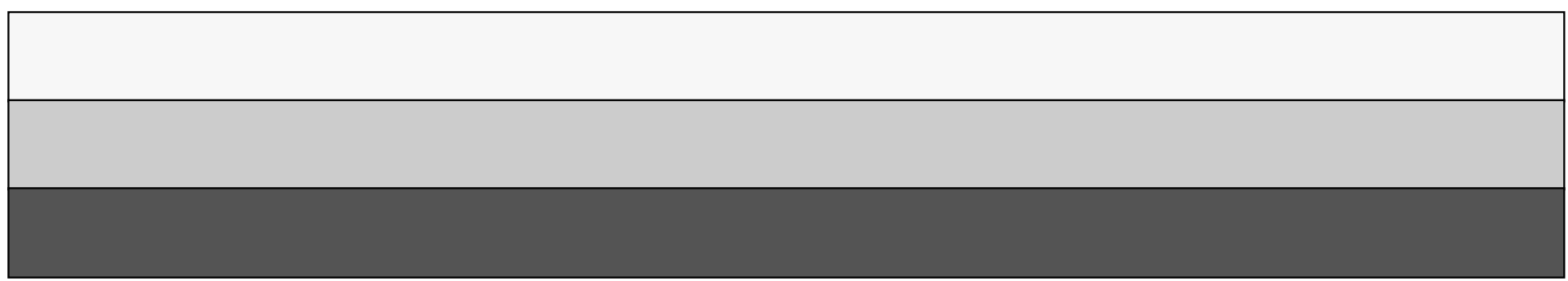

